ANUARIO DE ESTUDIOS MEDIEVALES 42/2, julio-diciembre de 2012, pp. 867-880

ISSN 0066-5061

doi:10.3989/aem.2012.42.2.11

\title{
MERCANTI E LIBRI DI CONTO NELLA TOSCANA DEL BASSO MEDIOEVO: LE EDIZIONI DI REGISTRI AZIENDALI DAGLI ANNI '60 DEL NOVECENTO A OGGI ${ }^{1}$
}

\author{
MERCHANT-BANKERS AND ACCOUNT BOOKS \\ IN LATE MEDIEVAL TUSCANY: \\ THE EDITIONS OF BUSINESS REGISTERS \\ FROM THE 1960'S TO TODAY
}

SERGIO TOGNETTI

Università degli Studi di Cagliari

\begin{abstract}
Riassunto: Nel panorama europeo la Toscana si segnala per il fatto di ospitare i più importanti archivi di società mercantili, bancarie e manifatturiere per la storia economica dei secoli XIII-XV. Soltanto tra Firenze e Prato si conservano circa 2.500 libri di conto, una cifra che eccede di gran lunga le disponibilità di tutto il resto dell'Italia e dell'Europa messe insieme. Il presente saggio fa il punto sulle edizioni di registri contabili aziendali pubblicati nell'ultimo mezzo secolo, con accenni anche ai decenni precedenti, in modo da mettere in risalto il ruolo ricoperto nella storia economica tardo-medievale dalle fonti contabili prodotte dai mercanti delle città toscane.
\end{abstract}

Parole chiave: Toscana; mercanti; libri contabili; archivi aziendali; storia economica; tardo-medioevo.

\begin{abstract}
In the European comparison Tuscany stands out by having the most important business archives (merchant, banking and manufacturing firms) for economic history of the later Middle Ages. The combined archives of Florence and Prato alone have around 2,500 account books, a figure greatly exceeding those available in the rest of Italy and Europe put together. The present paper considers the corporate account books published in the last 50 years, with reference, moreover, to the first half of the XXth century. The aim is to emphasize the role played by accounting sources written by the merchants of Tuscan towns in latemedieval economic history.
\end{abstract}

Keywords: Tuscany; merchants; account books; business archives; economic history; Late Middle Ages.

Per quanto attiene alle edizioni di fonti contabili, soprattutto di quelle che si configurano più propriamente come registri aziendali relativi alla Toscana del basso Medioevo, gli anni '60 del secolo scorso per molti aspetti rappresentano un momento

\footnotetext{
${ }^{1} \mathrm{Si}$ pubblica in forma rimaneggiata e ampliata il testo presentato al convegno Per la storia delle città toscane. Bilancio e prospettive delle edizioni di fonti dalla metà degli anni Sessanta a oggi (Firenze, 9-11 febbraio 2011), organizzato dal Centro Interuniversitario per la storia delle Città Toscane (CIRCIT) e della Deputazione di Storia Patria per la Toscana. Ringrazio Richard Goldthwaite e Francesco Guidi Bruscoli per l'aiuto fornito.
} 
di acquisizione e di riflessione matura, più che l'avvio di una nuova stagione di studi. I libri di conto prodotti in Toscana tra il XIII e la prima metà del XIV secolo divennero infatti oggetto di accurate indagini sin dal primo dopoguerra, per l'interesse vivissimo mostrato verso queste fonti sia dagli storici del commercio e della banca (curiosamente quasi tutti di formazione storico-giuridica) ${ }^{2}$, sia soprattutto dagli studiosi della lingua volgare toscana, i quali già negli anni precedenti il primo grande conflitto bellico si erano timidamente avvicinati a questa tipologia documentaria. Basterebbe soltanto pensare ai Testi fiorentini editi dallo Schiaffini nel 1926, tra i quali "campeggiano", da una parte, in terza riedizione (dopo quelle non del tutto soddisfacenti del Santini e del Monaci), i preziosi frammenti di un libro di conti tenuto a Bologna da banchieri fiorentini nell'anno 1211, miracolosamente salvatisi dalla dispersione come fogli di guardia di un codice del Digestum Novum cum glossa, conservato presso la Biblioteca Medicea Laurenziana di Firenze e databile tra fine XIII e inizio XIV secolo ${ }^{3}$; dall'altra le carte contabili di Cepparello di Dietaiuti da Prato (ovvero messer Ciappelletto, il sinistro e grottesco protagonista della prima novella del Decameron), già edite dal Paoli nel lontano $1885^{4}$.

Ma il vero salto di qualità arriva con i lavori condotti nei primissimi anni ’30 da Mario Chiaudano e Guido Astuti, entrambi fondamentalmente incentrati sui documenti mercantili relativi alle aziende senesi nel loro secolo d'oro, ovvero il Duecento ${ }^{5}$. Per approdare infine alle ricerche curate da Armando Sapori, con sontuose edizioni di libri contabili (soprattutto libri segreti) appartenuti alle grandi società mercantili-bancarie fiorentine della prima metà del Trecento ${ }^{6}$, veri e propri monumenti della storia economica, come ebbe a definirli Luigi Einaudi nel 1937 in una recensione ai Libri di commercio dei Peruzzi ${ }^{7}$; il quale Einaudi, da Presidente della Repubblica Italiana, volle anche scrivere la Premessa a I libri degli Alberti del Giudice pubblicati nel $1952^{8}$. Tanto i linguisti quanto gli storici economici erano decisamente animati dall'idea di studiare le origini di fenomeni grandiosi (l'affermazione di una lingua volgare nei decenni precedenti il suo maestoso exploit letterario da una parte, la nascita del capitalismo commerciale e bancario dall'altra), vagliando e pubblicando documenti straordinariamente precoci e praticamente introvabili (per questa altezza cronologica) in altri contesti archivistici, geografici e politici italiani, per non parlare dell'intero panorama europeo'.

Su un filone di ricerca già ben avviato e consolidato, a partire dal secondo dopoguerra, si inserirono le indagini di studiosi dalla spiccata personalità e originalità. Federigo Melis condusse vere e proprie campagne di scavo negli archivi fiorentini,

2 Armando Sapori si era laureato in diritto penale a Siena nel 1919 e, prima di ottenere la cattedra di storia economica presso l'Università di Ferrara nel 1932, era stato a lungo funzionario dell'Archivio di Stato di Firenze. Mario Chiaudano e Guido Astuti furono entrambi docenti di storia del diritto italiano.

${ }^{3}$ P. Santini, Frammenti di un Libro di banchieri, pp. 166-177; E. Monaci, Crestomanzia italiana, pp. 19-28; A. Schiaffini, Testi fiorentini, pp. 3-15.

4 C. Paoli, Documenti di Ser Ciappelletto, pp. 346-360; A. Schiaffini, Testi fiorentini, pp. 244-259.

${ }^{5}$ M. Chiaudano, Studi e documenti, in particolare si segnala la trascrizione integrale de Il libro delle fiere della Champagne, pp. 164-208; G. Astuti, Il libro dell'entrata e dell'uscita.

${ }^{6}$ A. Sapori, I libri di commercio; idem, I libri della ragione bancaria; idem, I libri degli Alberti. A questi dovrebbe aggiungersi un quaderno cartaceo conservato al Public Record Office di Londra con l'intestazione Tercius liber mercatorum de Ffriscobaldis, edito sempre da Sapori in calce al suo studio su La compagnia dei Frescobaldi, pp. 83-136.

${ }^{7}$ L. Einaudi, Un monumento, pp. 58-61.

${ }^{8}$ A. Sapori (ed.), I libri degli Alberti, pp. XIII-XVIII.

${ }^{9}$ Un ottimo bilancio del contesto europeo, datato 1956 ma ancor oggi in larga parte valido, è quello di R. de Roover, The development of accounting. 
pisani e soprattutto pratesi (penso ovviamente all'immenso fondo del mercante Francesco di Marco Datini) al fine di mettere in risalto una cesura fondamentale nell'evoluzione delle tecniche contabili, oltre che commerciali, bancarie e assicurative. Questo momento critico, individuato da Melis nella seconda metà del XIV secolo, fu interpretato in maniera diametralmente opposta rispetto alla visione consolidata da Sapori (e fatta propria da quasi tutta la medievistica del tempo): il pieno Trecento fu visto da Melis come un'epoca nella quale sarebbe maturata una risposta positiva alla sfida dei grandi cambiamenti strutturali determinati dai crac finanziari toscani dei primi decenni del secolo e ovviamente dalla Peste Nera ${ }^{10}$. Quale che sia la nostra interpretazione su drammatici rivolgimenti e trasformazioni del popolamento urbano e rurale, dell'economia e della società toscana, italiana ed europea, dal punto di vista della ragioneria tardo-medievale Melis aveva colto nel segno: un libro mastro di una grande società d'affari dell'inizio del Quattrocento, con i conti tenuti a sezioni contrapposte ("dare" sulla sinistra e "avere" sulla destra: in gergo del tempo si diceva alla "veneziana") e secondo una perfetta partita doppia, assomiglia enormemente di più ai libri contabili attuali di quanto non facciano i superstiti registri di un secolo prima.

Data la smisurata mole di documentazione che si trovava ora a maneggiare (non le decine di esemplari compulsate da Sapori, ma le centinaia tra mastri, quaderni di cassa, memoriali, libri di entrata e uscita, quaderni di mercanzie e tutta la lunga teoria di registri contabili minori), risultava impossibile per un solo studioso portare avanti edizioni di libri di commercio e, inoltre, più si procedeva verso il XV secolo e più le fonti si moltiplicavano e si standardizzavano: a questo punto la fatica dell'editore di libri di conto rischiava di divenire inversamente proporzionale al carattere di originalità delle nuove edizioni; come dimostra il fatto che i coniugi de Roover, esperti di documentazione mercantile toscana al pari di Melis (già nel 1934 Florence Edler de Roover aveva pubblicato un glossario relativo lessico mercantile italiano per i secoli XIII-XVI) ${ }^{11}$, non si cimentarono mai in edizioni integrali di libri contabili, e tuttavia pubblicarono saggi e volumi di grandissimo interesse sulla storia del commercio, della manifattura e della banca nella Toscana tardo-medievale ${ }^{12}$. L'esito di questo apparente cul de sac è che, dalla Storia della Ragioneria uscita nel 1950, passando per gli Aspetti della vita economica medievale del 1962, sino al volume postumo del 1975 sulla storia delle assicurazioni ${ }^{13}$, non c'è lavoro di Melis che non si sia avvalso di trascrizioni (talvolta anche copiose, per non dire "strabocchevoli" secondo un aggettivo caro alla prosa melisiana), ma manca nella sua bibliografia l'edizione completa e sistematica di uno o più registri: attività che per altro veniva commissionata ai suoi allievi, laureandi in Economia e Commercio delle Università di Pisa e di Firenze. Centinaia di libri segreti, mastri, memoriali, quaderni di ricordanze commerciali, ecc., dei secoli XIV-XVI furono trascritti nelle tesi di laurea e questa prassi non si interruppe neppure con la morte del Maestro, essendo stata fatta propria dai docenti dell'Istituto

${ }^{10}$ Sulla biografia umana e scientifica dello studioso si veda la lunga, ben argomentata e appassionata biografia di M. Del Treppo, Federigo Melis.

${ }^{11}$ F. Edler de Roover, Glossary.

12 Basterebbe soltanto citare il classico volume di R. de Roover, Il banco Medici e la raccolta postuma dei suoi saggi più importanti Business, banking; per F. Edler de Roover, oltre al citato glossario, si vedano gli studi condotti sull'industria serica: Andrea Banchi; Le sete lucchesi; L'arte della seta a Firenze.

${ }^{13}$ F. Melis, Storia della Ragioneria; idem, Aspetti della vita economica medievale; idem, Origini e sviluppi delle assicurazioni. La sterminata produzione scientifica di Melis è stata raccolta in una serie intitolata "Opere sparse di Federigo Melis", costituita da ben 7 volumi curati dai suoi allievi e usciti tra il 1984 e il 1991. 
di storia economica dell'Università di Firenze. Nondimeno, tutto questo patrimonio (la cui potenzialità è arricchita da preziosi indici di fondo) è spesso risultato di scarsissima utilità alla comunità degli studiosi, essendo le tesi di laurea virtualmente non consultabili, eppure spesso citate nelle opere di Melis e in quelle di alcuni affermati allievi, spesso senza specifici riferimenti ai documenti originali.

Sul versante degli storici della lingua occorre invece segnalare l'attività di un grande studioso quale Arrigo Castellani, probabilmente il maggior linguista italiano del '900 capace di comprendere e valorizzare l'importanza della documentazione mercantile duecentesca per la storia del volgare toscano delle origini. Da questo punto di vista i Nuovi testi fiorentini del Dugento del 1952 costituiscono un'opera a suo modo pionieristica e di grande valore scientifico, alla quale va idealmente accostata una raccolta esaustiva su tutti i testi toscani di carattere pratico antecedenti l'ultimo quarto del XIII secolo, pubblicata nel 1982 con un volume apposito di facsimili ${ }^{14}$.

A partire dagli anni '60, al contrario, l'interesse per l'edizione di fonti contabili aziendali toscane sembra scemare, almeno per gli storici economici. Mentre i linguisti a caccia di frammenti di volgare duecentesco e primo trecentesco hanno proceduto alla pubblicazione di qualsiasi esemplare di libro di conti (spesso anche di piccoli registri a carattere patrimonial-familiare $)^{15}$, valorizzando anche il panorama documentario relativo a città apparentemente minori (come Prato e Pistoia) ${ }^{16}$, gli studiosi di storia del commercio, dell'industria e della finanza si sono inevitabilmente spostati verso l'epoca posteriore alla Peste Nera, in virtù della maggiore abbondanza di fonti disponibili, non solo inedite ma anche inesplorate. Ma quanto più la documentazione ha permesso analisi quantitative e in certi casi addirittura seriali, tanto più improbo e avaro di prospettive originali è risultato lo sforzo di pubblicare libri di conti, di cui a stento si è riuscito a percepire il carattere di unicum. Tutto l'opposto potremmo dire per l'edizione dei libri di ricordanze familiari, oggetto di una serie notevole di pubblicazioni negli ultimi quattro-cinque decenni, fino alla recentissima fatica di Antony Molho e Franek Sznura ${ }^{17}$. Parenti alla lontana dei registri contabili aziendali, con i quali condividono aspetti non del tutto secondari (primo fra gli altri la presenza di numerose scritture deputate a tenere memoria del patrimonio di famiglia, della sua gestione più o meno quotidiana, delle entrate e delle uscite più rilevanti nel corso degli anni, delle rendite dei poderi e degli utili delle eventuali imprese), i libri di memoria familiare possiedono un fascino intrinseco che nessuno libro mastro di una banca potrà mai avere, oltre al fatto che se ne conserva qualche centinaia di unità per la Firenze dei secoli finali del Medioevo ${ }^{18}$, a fronte delle migliaia di libri contabili (solo per l'area fiorentina Goldthwaite ha stimato in circa 2.500 gli esemplari disponibili per i secoli XIII-XV, per arrivare alla cifra di 12 mila se includiamo anche il

14 A. Castellani, Nuovi testi fiorentini; idem, La prosa italiana. Nella sua infaticabile attività di editore di fonti mercantili medievali l'autore ha tuttavia manifestato una curiosa tendenza, certo motivata dai suoi interessi linguistici e filologici, a interrompere bruscamente le sue trascrizioni all'anno 1300 , e questo anche nel caso in cui una fonte superasse questa "fatidica" data.

${ }^{15}$ Come nei casi di M. Vitale, Il quaderno di ricordi e di P. Manni, Il libro del dare e dell'avere.

${ }^{16} \mathrm{Si}$ vedano, a titolo d'esempio, le trascrizioni e le analisi di libri contabili contenute in L. Serianni, Testi pratesi; P. Manni, Frammenti d'un libro di conti; P. Manni, Testi pistoiesi.

${ }^{17}$ A. Molho, F. Sznura, Brighe, affanni, volgimenti di stato.

18 G.M. Anselmi et al., La "memoria" dei mercatores, pp. 93-149, contavano un trentennio or sono 330 esemplari fiorentini editi (anche solo parzialmente e per piccoli brani), ma alcuni di questi debordano nel XVI secolo se non addirittura nel XVII, altri non sono propriamente libri di ricordanze familiari, bensì diari e cronache cittadine, per non parlare di quei registri che in realtà non hanno tutti i crismi necessari per essere considerati libri di famiglia poiché sono più propriamente libri di conto "personali", cioè non intestati alle aziende, ma comunque dall'indole tipica del registro contabile. 
Cinquecento $)^{19}$. Un discorso in parte simile riguarda la pubblicazione del carteggio mercantile, che negli ultimi anni ha conosciuto un piccolo boom editoriale (in gran parte incentrato sull'immenso deposito datiniano ora consultabile anche on-line $)^{20}$, finendo quasi per surclassare la fonte contabile. Tuttavia, per quanto apparentemente più avvincenti dei libri aziendali, le lettere dei mercanti non sempre permettono allo studioso di entrare nel vivo della gestione di un'impresa, di evidenziare l'evoluzione di una strategia aziendale e meno che mai di ricostruirne la redditività.

Le edizioni integrali di libri mastri e registri contabili in generale hanno quindi subito, nell'ultimo mezzo secolo, un processo di selezione molta alta, ispirata fondamentalmente a due criteri strettamente interdipendenti: il carattere di eccezionalità della fonte e la sua più o meno relativa precocità. In quest'ottica mi sembra che debbano essere segnalate per la loro rilevanza almeno sei imprese editoriali: Il libro vermiglio di corte di Roma e di Avignone del segnale del C della compagnia fiorentina di Iacopo Girolami, Filippo Corbizzi e Tommaso Corbizzi, edito da Mario Chiaudano nel 1963 senza uno studio storico introduttivo ${ }^{21}$; il Libro giallo della compagnia dei Covoni pubblicato da Sapori nel 1970, con una lunga presentazione del manoscritto da parte di Giulio Mandich ${ }^{22}$; gli ormai celebri Documenti per la storia economica dei secoli XIII-XVI, antologia di fonti mercantili (soprattutto toscane) curata da Melis nel 1972²3. Il libro Giallo di Ginevra della compagnia fiorentina di Antonio della Casa e Simone Guadagni edito da Michele Cassandro nel 1974 con un ampio studio introduttivo $^{24}$; i Due libri mastri degli Alberti pubblicati nel 1995, con trascrizione curata da Enzo Settesoldi e saggi di Richard Goldthwaite e Marco Spallanzani' ${ }^{25}$; infine l'antologia di numerosi ma frammentari registri superstiti dell'archivio della compagnia Gallerani-Fini conservatosi a Gand, antologia assai egregiamente curata dalla storica della lingua Roberta Cella nel 200926.

Come è di tutta evidenza, tra la metà degli anni ' 70 e la metà degli anni '90 c'è un vuoto notevole, testimonianza non marginale, a mio avviso, della progressiva caduta d'interesse non solo per l'edizione di fonti contabili aziendali, ma per la storia dei mercanti medievali italiani nel suo complesso. Le due successive pubblicazioni, per quanto assai importanti, sono essenzialmente il frutto di ritrovamenti straordinari in archivi non toscani.

Vediamo sommariamente alcuni degli elementi sostanziali delle iniziative editoriali sopra accennate. Il libro vermiglio tenuto presso la corte pontificia di Avignone dalla compagnia bancaria dei Girolami-Corbizzi tra 1332 e 1337, e conservato nell'Archivio Vaticano di Roma, è forse il testo meno rilevante e quindi meno citato dagli storici, anche per la reticenza di Chiaudano a fornire un'introduzione storica adeguata all'esemplare pubblicato, solo in parte compensata da un breve ma acuto

${ }^{19}$ R.A. Goldthwaite, The economy, p. XII.

${ }^{20} \mathrm{Si}$ vedano a titolo di esempio le seguenti pubblicazioni: L. Frangioni, Milano fine Trecento; G. Nigro, Mercanti in Maiorca; A. Castellani, I. Del Punta, Lettere dei Ricciardi; A. Orlandi, Mercanzie e denaro. A riprova di ciò è interessante osservare come nel grosso e miscellaneo volume $\mathrm{G}$. Nigro, Francesco di Marco Datini, quasi tutti i saggi si basino più sull'utilizzo del carteggio che non sul vaglio della documentazione contabile.

${ }^{21}$ M. Chiaudano, Il libro vermiglio.

22 A. Sapori, Libro giallo della compagnia dei Covoni.

${ }^{23}$ F. Melis, Documenti.

${ }^{24}$ M. Cassandro, Il libro Giallo di Ginevra.

25 R.A. Goldthwaite, E. Settesoldi, M. Spallanzani, Due libri mastri.

${ }^{26}$ R. Cella, La documentazione. 
saggio di Sapori e da alcuni significati rilievi di Melis nei suoi Documenti ${ }^{27}$. Invero, nonostante il fatto che il mastro faccia parzialmente luce su interessanti aspetti di natura finanziaria per l'Avignone papale a un'altezza cronologica relativamente precoce (siamo negli anni a cavallo tra il pontificato di Giovanni XXII e quello di Benedetto XII), le potenzialità del manoscritto contabile sono in una certa misura inficiate dalla circostanza che la compagnia Girolami-Corbizzi (certamente non delle più importanti nel panorama fiorentino dell'epoca) si sciolse dopo appena due anni dal suo avvio per una serie di liti e gravi discordie tra i soci, uno dei quali finì anche in prigione per frode. Così, su 227 carte disponibili originariamente ne furono utilizzate solo 87. Inoltre, una piccola parte del registro fu dedicata a procedure di liquidazione; e a questo si devono aggiungere poste contabili concernenti spese personali e di famiglia dei soci. Nondimeno rimane da segnalare una discreta attività di prestito documentata da qualche decina di conti intestati a ecclesiastici, funzionari laici e religiosi della corte pontificia, nobili, castellani, cavalieri e cortigiani soprattutto, com'è ovvio, di origine francese e provenzale; nonché alcuni significativi riferimenti a una ventina di compagnie d'affari fiorentine presenti ad Avignone negli anni '30 del XIV secolo.

Il libro grande di debitori e creditori con la coperta di cuoio colorata di giallo appartenuto alla compagnia fiorentina dei Covoni copre l'arco cronologico 1336-1339. Il registro, composto da oltre 280 carte (con qualche foglio mancante), si trova all' archivio di stato di Firenze, all'interno del fondo del Bene: il più importante deposito fiorentino (e direi europeo) di materiale aziendale (manifattura, commercio e banca) per l'arco cronologico compreso tra la seconda metà del Duecento e il 1370 circa, quando il testimone ideale passa al colossale fondo datiniano di Prato. L'azienda in questione non è minimamente paragonabile alle grandi società d'affari operanti sullo scenario internazionale nella prima metà del XIV secolo, già studiate ampiamente dallo stesso Sapori. Già solo per questo il mastro dei Covoni ha un suo specifico interesse, non essendo il prodotto di una ristrettissima élite mercantile e finanziaria, ma lo specchio (forse anche più rappresentativo) di un milieu fiorentino costituito da tante imprese commerciali e bancarie di medio livello. Il contenuto del libro di conti rivela inoltre alcune particolarità che ne fanno a suo modo un unicum: in esso, infatti, troviamo ampiamente documentate operazioni bancarie e finanziarie che sino all'edizione di questa fonte ci saremmo aspettati di trovare solo nella documentazione contabile degli ultimi decenni del Trecento. In primo luogo, l'uso sistematico e assai disinvolto della lettera di cambio, non solo per i suoi impieghi primari (trasferimenti di valuta su piazze straniere per le esigenze più varie: pagamenti a fornitori esteri, rimesse per ricavi di merci vendute fuori piazza, ecc.), ma anche per le attività di prestito speculativo. A centinaia si contano le poste contabili relative a lettere di cambio che fanno letteralmente la spola tra Firenze e Venezia; per non parlare dei negozi cambiari del tutto fittizi, cioè i "cambi secchi", regolati senza alcun effetto bancario ma semplicemente sulla base delle quotazioni delle valute fiorentine e veneziane, manipolate ad arte dalla comunità dei banchieri e dei cambiavalute per avvantaggiare in maniera metodica il datore della valuta, ovvero il prestatore di una somma. Una prassi, conosciuta in età tardo-medievale con l'espressione idiomatica "stare in su' cambi", che era già stata evidenziata da Raymond de Roover sulla base di documentazione primo-quattrocentesca conservata nell'archivio della Fraternita dei Laici di Arezzo (libri mastri del mercante aretino Lazzaro Bracci) ${ }^{28}$ e sulla quale ha poi fatto pienamente luce Reinhold Mueller in una monografia dedicata alla banca a Venezia

${ }^{27}$ A. Sapori, Una compagnia di prestatori; F. Melis, Documenti, pp. 44, 52, 58, 59, 71, 85, 98.

${ }^{28}$ R. de Roover, Cambium ad Venetias. 
nel tardo Medioevo ${ }^{29}$. L'altro aspetto, forse un po' sottovalutato, è quello relativo a forme di pagamenti e adempimenti di obbligazioni, il cui formulario standardizzato ricorda molta da vicino quello enucleato da Melis per la registrazione contabile di ordini di pagamento scritti (ovvero mandati all'incasso e assegni bancari veri e propri), in riferimento però all'attività della banca a Pisa, e più in generale in Toscana, della seconda metà del Trecento ${ }^{30}$. Può darsi che il grande storico della ragioneria non abbia avuto né tempo né modo di confrontare le sue acquisizioni, maturate sui registri contabili pisani e su quelli datiniani, con quelle emerse dal libro dei Covoni, essendo egli scomparso alla fine del 1973. Anche se rimane il sospetto che lo tenesse distante da tale preoccupazione il marchio "saporiano" sull'impresa editoriale ${ }^{31}$. Ma questa precocissima ed eccezionale prassi bancaria che caratterizza il registro sarebbe quasi sicuramente sfuggita a Sapori, che era certamente più storico di Melis, ma assai meno ferrato in questioni di contabilità medievale. Per questo il vero studio introduttivo al testo venne affidato a Mandich ${ }^{32}$, mentre Sapori si accontentò di una premessa sulla storia della famiglia Covoni in età basso-medievale con qualche spunto sulle epoche successive ${ }^{33}$. Chiunque abbia letto il saggio di Mandich, lungo, approfondito e competente $^{34}$, ma anche infarcito di termini giuridici latini e di esasperati tecnicismi inavvicinabili per un neofita di contabilità medievale, sintatticamente assai tormentato e molto debole per quanto riguarda l'inquadramento della fonte nel contesto del più generale dibattito storiografico, riesce a spiegarsi perché a questa pubblicazione non è stata data tutta l'importanza che meritava. Lo stesso Sapori se ne rese evidentemente conto quando, nella ricordata premessa, ebbe modo di affermare, con sottile (e forse perfida) ironia:

ho lanciato un appello a un mio carissimo amico, studioso di estrema serietà e specializzato in materia, il prof. Giulio Mandich. Non solo l'amico ha risposto alla mia richiesta, ma si è sottoposto ad uno sforzo che se avessi saputo tale non gli avrei chiesto ${ }^{35}$

L'antologia documentaria curata da Melis poco prima della sua scomparsa ha uno scopo semplicemente e altamente didattico: di fatto è una sorta di sontuoso monumento che l'autore ha voluto erigere ai suoi anni di magistero pisano e fiorentino quale docente di storia economica della Toscana basso-medievale e rinascimentale. Il volume ha una lunga introduzione metodologica nella quale vengono esposte e commentate le varie tipologie in cui Melis amava suddividere le fonti prodotte direttamente dai mercanti e dalle loro aziende: carteggio comune, carteggio specializzato, scritture private, libri di contabilità sintetica e analitica, manuali di preparazione e consultazione, con due sezioni specifiche dedicate ai documenti incentrati sull'attività bancaria e sulle manifatture tessili. Quindi si passa all'illustrazione assai dettagliata dei numerosi esemplari con tanto di trascrizione e riproduzione fotostatica dell'originale.

${ }^{29}$ R.C. Mueller, The Venetian money market, in particolare pp. 288-355. Su questi aspetti mi permetto di rimandare anche alla mia recensione: I mercanti-banchieri fiorentini.

${ }^{30}$ F. Melis, Note di storia della banca, in particolare i capp. III e IV.

${ }^{31}$ Almeno così mi presentò la cosa, una quindicina di anni fa, Bruno Dini, allievo di Melis sin dal periodo del suo magistero pisano.

${ }^{32}$ G. Mandich, Per una ricostruzione.

33 A. Sapori, Premessa.

${ }^{34}$ R.A. Goldthwaite, The economy, p. 217 n. 31, definisce giustamente il saggio "a definitive analysis of the various forms of the bill as seen though the banker's account".

${ }^{35}$ A. Sapori, Premessa, p. XIX.

ANUARIO de Estudios MEdievales, 42/2, julio-diciembre 2012, pp. 867-880 ISSN 0066-5061, doi:10.3989/aem.2012.42.2.11 
Il libro giallo della compagnia mercantile-bancaria fiorentina dei della Casa-Guadagni ha bisogno di qualche spiegazione in più. Il registro, uno splendido esempio di perfetta partita doppia con conti tenuti a sezioni contrapposte, cioè alla veneziana, è il primo di tre libri mastri tenuti a Ginevra tra gli anni ' 50 e gli anni ' 60 del Quattrocento e conservatisi nel formidabile fondo Estranei presente nell'Archivio dell'Ospedale degli Innocenti di Firenze, il cui nuovo inventario, curato da Lucia Sandri con il finanziamento del Max-Plank-Institut, è ora disponibile agli studiosi in un comodo e agevole formato on-line ${ }^{36}$. Di fatto i tre manoscritti sono l'unica testimonianza aziendale di prima mano dell'attività degli uomini d'affari fiorentini (e non solo) alle fiere internazionali di Ginevra, il più grande raduno commerciale e la principale sede di transazioni finanziarie e bancarie del continente europeo durante tutta la prima metà del XV secolo e fino all' ascesa di Lione negli anni ' $60^{37}$. Da questo osservatorio privilegiato possiamo quindi cogliere il flusso delle merci fiorentine verso Ginevra, soprattutto raffinati drappi di seta destinati alla migliore clientela dell'Europa centrale e occidentale, ma ancora una volta un pullulare di attività finanziarie legate ai traffici di lettere di cambio e speculazioni sulle valute internazionali. Il curatore dell'edizione, a suo tempo un allievo di Melis, ha realizzato una pregevole edizione utilizzando criteri che, come spiegherò in chiusura, rimangono a mio avviso i migliori, almeno se il punto di vista adottato è quello di uno storico dell'economia e dell'impresa.

Il quinto caso si riferisce a due grossi libri mastri appartenuti a una delle compagnie Alberti operanti tra 1348 e 1358 . La società si configura come una filiazione aziendale proveniente da quella compagnia Alberti del primo Trecento, i cui libri contabili, conservati presso la Biblioteca Nazionale Centrale di Firenze, erano stati editi da Sapori e ampiamente studiati anche da Raymond de Roover ${ }^{38}$. Dei due nuovi codici si sono conservate alcune corpose sezioni che furono rinvenute da Settesoldi nell'abitazione genovese del conte Benedetto Nerozzo Alberti (ora Archivio Gaslini Alberti di Genova). I registri sono tenuti in partita doppia ma a sezione sovrapposte, con la sezione dare che occupava tutta la prima parte dei codici e quella avere la seconda. Le perdite hanno colpito soprattutto l'avere e in piccola parte il dare. Nonostante le gravi mutilazioni, i manoscritti hanno una notevole rilevanza sul piano storico: come affermano Goldthwaite e Spallanzani si tratta degli esemplari più antichi di mastro, ovvero di libri debitori e creditori, di una compagnia di rango internazionale, considerando quindi i libri dei Girolami-Corbizzi e dei Covoni non espressione di società d'affari di alto livello. Inoltre, il primo dei due mastri albertiani è l'unico libro contabile appartenuto a una compagnia che (...) continuò ad essere mantenuta attiva durante i giorni in cui la Peste Nera stava flagellando Firenze e tutta l'Europa ${ }^{39}$. Ma soprattutto ciò che colpisce è la persistenza, oltre l'epoca segnata dai grandi fallimenti dei Bardi, dei Peruzzi e degli Acciaiuoli, di un modello organizzativo ancora tradizionale e ben lontano dagli esempi tardo-trecenteschi studiati da Melis. Le società Alberti ancora negli anni '50 del secolo avevano una strategia d'affari del tutto in linea con quella delle compagnie di Calimala del primo Trecento, imperniate sull'import-export dei così detti panni franceschi e sulla mancata separazione, a livello giuridico, tra la

${ }^{36}$ http://www.istitutodeglinnocenti.it/culturali/inventario.jsf.

37 Oltre al lungo saggio introduttivo di Michele Cassandro al primo libro mastro, si veda anche del medesimo autore Banca e commercio. L'opera di riferimento generale per la storia delle fiere di Ginevra nel '400 è quella di J.F. Bergier, Genève. Ampi riferimenti al tema sono contenuti anche in R. de Roover, Il banco Medici.

${ }^{38} \mathrm{R}$. de Roover, The story of the Alberti.

${ }^{39}$ R.A. Goldthwaite, E. Settesoldi, M. Spallanzani, Due libri mastri, p. XXIV. 
casa madre fiorentina e le filiali all'estero; una prassi che era costata molto cara alle "multinazionali" toscane, quando il crac anche di una sola succursale in terra straniera aveva comportato il fallimento a catena di tutta l'impresa, in virtù della responsabilità solidale e illimitata dei soci. Anche dal punto di vista delle operazioni bancarie $i$ due mastri degli Alberti (...) rappresentano non tanto un'apertura verso la nuova realtà quanto la continuazione, ancora negli anni 1350-60, del vecchio mondo ${ }^{40}$.

L'ultima recentissima opera di pubblicazione di fonti mercantili toscane, e segnatamente (ma non solo) di libri di conto, è quella di Roberta Cella, storica della lingua. Diciamo subito che i suoi studi archivistici nell'archivio di Gand e l'antologia di fonti da lei proposta sono entrambi destinati a lasciare più di un segno nella storiografia sugli uomini d'affari toscani nell'Europa tra Due e Trecento (almeno questo è l'auspicio del sottoscritto), perché in questo caso non si parla di libri contabili fiorentini, ma di un vero e proprio archivio di una compagnia d'affari senese, quella dei Gallerani-Fini. Con una certa dose di giustificata enfasi retorica la curatrice arriva a definire questo riscoperto archivio aziendale una Pompei documentaria medievale $e^{41}$.

Nell'ottobre del 1309 i fratelli Bartolomeo e Tommaso Fini, già soci della compagnia senese dei Gallerani e tesorieri del conte di Fiandra Roberto III di Béthune, vennero accusati di malversazione e quindi arrestati. Bartolomeo fu giudicato colpevole e giustiziato, Tommaso riuscì a scappare da Bruges e rifugiarsi in Francia, probabilmente con l'appoggio del re Filippo IV il Bello. Le merci e la documentazione amministrativa dei due uomini d'affari senesi vennero confiscate, insieme ai libri contabili della società dei Gallerani che, dalle filiali di Parigi e Londra, continuava ad appoggiarsi ai Fini per i traffici con Bruges e le città fiamminghe. Conservatosi miracolosamente nell' archivio comitale, con lungo soggiorno in un granaio, il dossier Gallerani-Fini, pur se sottoposto all'inevitabile pluri-secolare deperimento dovuto a topi, tarli e muffa, fu infine depositato nel Rijksarchief di Ghent alla metà del XIX secolo. Molti decenni fa, prima Georges Bigwood (scomparso nel 1930), poi Armand Grunzweig ne avevano valorizzato solo una piccola parte, pubblicando appena due libri di conto, di cui uno assai mutilo e lacunoso ${ }^{42}$. Avvicinatasi, a suo dire quasi per caso, ai restanti faldoni non ancora inventariati, Cella ha fornito agli storici uno strumento indispensabile per la comprensione di questo antico fondo che getta uno squarcio di luce impressionante sull'epopea dei mercanti-banchieri senesi di inizio Trecento, e si è dimostrata particolarmente brillante nell' approfondire la storia degli archivi aziendali basso-medievali (molti dei documenti in questione sono stati da lei stessa inventariati per la prima volta), coltivando una spiccata sensibilità per lo studio delle tecniche commerciali e delle registrazioni contabili.

L'importanza del deposito Gallerani-Fini, è ampiamente illuminata da una nutritissima selezione di exempla, preceduta a sua volta da una raffinatissima analisi codicologica, paleografica e linguistica. Sul piano storico economico la curatrice, giustamente, tende a sottolineare la complessità di un fondo che per consistenza e varietà della documentazione risulta quasi eccezionale, considerando anche l'altezza cronologica in questione: si tratta di 30 libri di conto di vari formati e tipologia (di cui uno addirittura in francese), 7 lettere, 36 annotazioni contabili, 4 scritture non contabili e 4 attergati (secondo la terminologia adottata dalla Cella), appartenuti alle filiali Gallerani-Fini di Parigi, Champagne, Provenza, Londra, Fiandre e Italia e cronologicamente concentrati tra il 1304 e il 1309. Alcuni registri contabili paiono già avere tutti i requisiti necessari per certificare la conoscenza e l'applicazione della partita

${ }^{40}$ Ibidem, p. CXIV.

${ }^{41}$ R. Cella, La documentazione, p. 8.

${ }^{42} \mathrm{G}$. Bigwood, Les livres des comptes. 
doppia. Del pari non mancano le attestazioni di negozi finanziari imperniati su lettere di cambio, come nel caso del libro di entrata e uscita della filiale di Londra del biennio 1304-1305 che ospita abbondanti testimonianze di cambi tra Lombard Street e le fiere della Champagne. Veramente eccezionale è poi l'originale di un effetto cambiario spiccato da Londra sulla corte pontificia di Roma, datata 5 ottobre 1305: per quello che mi consta il più antico esemplare di lettera di cambio conservato in Europa ${ }^{43}$.

Altrettanto interessante è il fatto che queste fonti, pur concentrate in un arco temporale ristretto, siano capaci di fornire informazioni su fenomeni di grande portata per la storia dell'organizzazione commerciale nell'Europa nord-occidentale del primo Trecento: per esempio emerge chiaramente il passaggio delle consegne tra il "vecchio" polo principale degli scambi internazionali (le fiere della Champagne) e il nuovo emporio di Bruges, dove le compagnie toscane porranno importanti succursali (tra cui quella dei Gallerani-Fini nel 1305). Ma già prima di questa data, nella strategia aziendale degli uomini d'affari senesi (ma evidentemente anche fiorentini, lucchesi, piacentini, astigiani e italiani in generale), l'attività del commercio di fiera nella Champagne era quasi totalmente diretta dalle sede parigina e lì risiedevano i maggiori "mercatanti" nel regno di Francia. E anche la composita nazione dei "lombardi" di Londra, nella quale i toscani erano parte non marginale, doveva essere assai nutrita e ben strutturata sul piano istituzionale, se il primo maggio del 1304 la filiale londinese dei Gallerani-Fini pagò 6 soldi e 8 denari di sterlini ala comunità dei lonbardi dimoranti a Londra per nostra parte dele spese che ssi feciero nel procacciare la dilivrança del tagliagio che n'avieno tagliati i borghesi di Londre ${ }^{44}$.

In sostanza, la pubblicazione fotografa come meglio non potrebbe l'apogeo del mondo affaristico senese prima della drammatica recessione trecentesca, ed è impreziosita da un ricco e intelligente apparato di indici fatto degli antroponimi e dei toponimi, delle cose notevoli e dei testi antichi, dei fenomeni linguistici e delle forme citate.

L'unica, modesta, osservazione critica che si potrebbe rivolgere alla Cella riguarda la definizione del fondo Gallerani-Fini come del più importante archivio aziendale basso-medievale antecedente quello di Francesco Datini ${ }^{45}$. Infatti, è solo parzialmente vero che di nessuna compagnia d'affari toscana, italiana ed europea si siano conservati tanti documenti (e soprattutto per una tipologia così varia e articolata) per la prima metà del XIV secolo, dato che il già citato fondo Del Bene depositato presso l'Archivio di Stato di Firenze ospita decine di registri contabili a partire dalla seconda metà del XIII secolo, prodotti e conservati da almeno quattro-cinque generazioni di uomini d'affari. Inoltre, molta della documentazione appartenuta ai Gallerani-Fini appare (per esplicita ammissione della curatrice) assai lacunosa e frammentata. Gli stessi libri di conto contengono pochissime decine di carte (ma talvolta i fogli superstiti si contano sulle dita di una mano) a fronte delle centinaia di cui erano originariamente composti. Forse non abbastanza per poterli mettere a confronto con i pochi ma corposi libri segreti e libri mastri appartenuti ai Peruzzi, ai Gianfigliazzi, agli Alberti, ai Gianfigliazzi, ai Covoni, solo per citare le aziende mercantili fiorentine del primo Trecento oggetto degli studi e delle edizioni di Sapori. Ma la vicenda editoriale ha già alimentato dibattiti e nuovi studi sul mondo mercantile senese dei decenni a cavallo del 1300 e soprattutto sulle tortuose vicende archivistiche che spesso hanno segnato a Siena la scomparsa di un patrimonio di scritture contabili che doveva essere imponente ${ }^{46}$.

\footnotetext{
${ }^{43}$ R. Cella, La documentazione, pp. 290-291.

${ }^{44}$ Ibidem, p. 242.

45 Ibidem, pp. 9-10.

${ }^{46}$ G. Piccinni, Libri di contabilità; eadem, Sede pontificia.
} 
Nel contesto toscano, tuttavia, il convitato di pietra non è Siena, ma Lucca. È bene precisare, infatti, che la gran massa di libri contabili toscani di età bassomedievale si concentra nel periodo posteriore all'epoca dei grandi fallimenti trecenteschi e alla Peste Nera; e questo vale non solo per gli archivi di Firenze e di Prato, ma anche di Pisa e di Arezzo, i cui fondi documentari mercantili tardo-trecenteschi e primo-quattrocenteschi furono ben valorizzati dagli studi di Melis e dai suoi allievi, pur senza produrre edizioni integrali di registri aziendali. Ebbene, a questa altezza cronologica Siena non solo non era più una potenza mercantile e bancaria di livello internazionale, ma il suo ceto dirigente stava rapidamente dirottando capitali, energie umane e sistemi di valori versa la rendita immobiliare, gli uffici pubblici e i titoli del debito pubblico: rimasero solo le banche, ma con un raggio d'azione assai più ristretto rispetto a quello di un secolo prima ${ }^{47}$. Anche così è singolare la scomparsa di presso che tutti gli archivi aziendali tre-quattrocenteschi, ma che dire allora di Lucca, i cui imprenditori serici vendevano velluti e broccati alle corti di mezza Europa e i cui mercanti-banchieri, come ha di recente dimostrato un bel volume di Laura Galoppi$\mathrm{ni}^{48}$, dominavano la piazza di Bruges all'epoca dei magnifici e munifici duchi di Borgogna? Nonostante gli uomini d'affari lucchesi non siano mai scomparsi dalla scena internazionale fino al pieno Cinquecento (furono loro a mettere in piedi la manifattura della seta prima a Lione e poi nella "eretica" Ginevra), per l'ultimo secolo e mezzo del Medioevo i registri contabili delle loro aziende sono di fatto latitanti. Il problema, storicamente e archivisticamente parlando, non è quindi la povertà documentaria di Siena, bensì l'eccezionalità fiorentino-pratese con le sue appendici pisane e aretine: l'incredibile deserto di documentazione aziendale in metropoli mercantili medievali, come Venezia e Genova, o in una città manifatturiera per eccellenza come Milano, è la dimostrazione e contrario del fenomeno. E una recente pubblicazione di Enrique Cruselles per la città di Valencia, ma con un meritorio approccio comparativo che abbraccia l'intera Europa basso-medievale, non fa che confermare questo fenomeno ${ }^{49}$. In conclusione emerge abbastanza chiaramente che, in un panorama comunque non particolarmente esaltante per quanto riguarda l'interesse verso la storia del commercio, delle manifatture e della banca nell'Italia basso-medievale (un fenomeno ormai pluri-decennale su cui si innesta il progressivo abbandono della storiografia anglosassone, e segnatamente statunitense, per la storia dell'Italia medievale tout court), le edizioni di libri di conto toscani non sono materia all'ordine del giorno, a meno di ritrovamenti straordinari, quali quelli illustrati. Nel caso avvenisse, mi permetto di suggerire agli editori di rispettare la volontà dei ragionieri medievali toscani, facendo mia una vecchia critica di Melis ai lavori di Castellani ${ }^{50}$. Quasi tutte le edizioni citate (comprese quelle di Sapori), ma in particolare quelle di Castellani e dei suoi affermati allievi (penso a Luca Serianni e a Paola Manni), così come quelle della Cella, costituiscono per lo storico dell'economia opere di non immediata e agevole fruibilità. Intanto, per motivi tipografici non è quasi mai possibile rispettare la cartulazione originale, un difetto che in un libro di conti caratterizzato da rimandi interni continui, con una numerazione originale delle carte spesso impostata non recto-verso ma a facciate sinistra-destra, non è di lieve entità; se poi l'edizione è condotta da un linguista, il suo scrupolo filologico tende inevitabilmente a riempire la pagina di continue parentesi

47 Sulle vicende dell'attività bancaria a Siena sullo scorcio dell'età medievale mi permetto di rinviare a S. Tognetti, "Fra li compagni palesi”. Il saggio era già in bozze quando è uscito il volume di G. Piccinni, Il Banco dell'ospedale.

${ }^{48}$ L. Galoppini, Mercanti toscani, in particolare i capp. III-V e XI.

${ }^{49}$ E. Cruselles Gómez, Los comerciantes valencianos.

${ }^{50}$ F. Melis, Osservazioni preparatorie, p. 229. 
tonde per segnalare al lettore lo scioglimento di segni abbreviativi, rendendo assai faticosa la lettura. Ma il punto fondamentale sta nel mancato uso delle tabulazioni per la trascrizione delle cifre che nei libri di conto sono sbalzate a destra et pour cause: soprattutto colui che teneva il libro mastro doveva avere ben chiaro quali importi sommare per chiudere i conti della carta! Se l'ammontare relativo a ogni singola scrittura viene posto anonimamente e mimeticamente al termine e non a fianco della posta contabile, con una procedura che filologicamente mi pare non difendibile oltre che dannosa per la lettura, lo storico economico sarà costretto a un inutile sforzo supplementare per ricostruire, con maggior fatica del dovuto, attività e giro d'affari di un'impresa. E un lettore non avvezzo a queste fonti potrebbe trarre l'erronea conclusione che i conti dei mercanti toscani erano tenuti in maniera poco chiara e poco ordinata.

\section{BIBLIOGRAFIA CITATA}

Anselmi, Gian Mario; Pezzarossa, Fulvio; Avellini, Luisa, La "memoria" dei mercatores. Tendenze ideologiche, ricordanze, artigianato in versi nella Firenze del Quattrocento, Bologna, Patron, 1980.

Astuti, Guido, Il libro dell'entrata e dell'uscita di una compagnia mercantile senese del secolo XIII (1277-1282), Torino, Lattes, 1934.

Bergier, Jean-François, Genève et l'économie européenne de la Renaissance, Paris, SEVPEN, 1963.

Bigwood, Georges, Les livres des comptes des Gallerani, Bruxelles, Académie Royale de Belgique, 1961-1962.

Cassandro, Michele, Banca e commercio fiorentini alle fiere di Ginevra nel XV secolo, "Rivista Storica Svizzera" 26 (1976), pp. 567-611.

Cassandro, Michele, Il libro Giallo di Ginevra della compagnia fiorentina di Antonio della Casa e Simone Guadagni, 1453-1454, Prato, Istituto Internazionale di Storia Economica "F. Datini", 1976.

Castellani, Arrigo (ed.), Nuovi testi fiorentini del Dugento, Firenze, Sansoni, 1952.

Castellani, Arrigo (ed.), La prosa italiana delle origini, I. Testi toscani di carattere pratico, Bologna, Patron, 1982.

Castellani, Arrigo; Del Punta, Ignazio (eds.), Lettere dei Ricciardi di Lucca ai loro compagni in Inghilterra (1295-1303), Roma, Salerno Editrice, 2005.

Cella, Roberta, La documentazione Gallerani-Fini nell'Archivio di Stato di Ghent (1304-1309), Firenze, Sismel - Edizioni del Galluzzo, 2009.

Chiaudano, Mario, Studi e documenti per la storia del diritto commerciale italiano nel sec. XIII, Torino, Istituto giuridico della R. Università, 1930.

Chiaudano, Mario (ed.), Il libro vermiglio di corte di Roma e di Avignone del segnale del C della compagnia fiorentina di Iacopo Girolami, Filippo Corbizzi e Tommaso Corbizzi, 1332-1337, Torino, Vincenzo Bona, 1963.

Cruselles Gómez, Enrique, Los comerciantes valencianos del siglo XV y sus libros de cuentas, Valencia, Universitat Jaume I, 2007.

Del Treppo, Mario, Federigo Melis, storico, in Studi in memoria di Federigo Melis, Napoli, Giannini, 1978, vol. I, pp. 1-87.

De Roover, Raymond, Business, banking, and economic thought in late Medieval and early Modern Europe, Chicago, University of Chicago Press, 1974.

De Roover, Raymond, Cambium ad Venetias: contribution to the history of foreign exchange, in De Roover, Raymond, Business, banking, and economic thought in late Medieval and early Modern Europe, Chicago, University of Chicago Press, 1974, pp. 239-259. 
De Roover, Raymond, Il banco Medici dalle origini al declino (1397-1494), [trad. it.], Firenze, La Nuova Italia, 1970.

De Roover, Raymond, The development of accounting prior to Luca Pacioli according to the account books of medieval merchants, in De Roover, Raymond, Business, banking, and economic thought in late Medieval and early Modern Europe, Chicago, University of Chicago Press, 1974, pp. 119-180.

De Roover, Raymond, The story of the Alberti company of Florence, 1302-1348, as revealed in its account books, in De Roover, Raymond, Business, banking, and economic thought in late Medieval and early Modern Europe, Chicago, University of Chicago Press, 1974, pp. 39-84.

Edler de Roover, Florence, Glossary of Mediaeval terms of business, Italian series, 1200-1600, Cambridge (Mass.), The Mediaeval Academy of America, 1934.

Edler de Roover, Florence, Andrea Banchi setaiolo fiorentino del Quattrocento, "Archivio Storico Italiano" 150 (1992), pp. 877-963.

Edler de Roover, Florence, Le sete lucchesi, Lucca, Istituto storico lucchese, 1993.

Edler de Roover, Florence, L'arte della seta a Firenze nei secoli XIV e XV, ( S. Tognetti ed.), Firenze, Olschki, 1999.

Einaudi, Luigi, Un monumento di storia economica, "Rivista di storia economica" 2 (1937), pp. 58-61.

Frangioni, Luciana, Milano fine Trecento. Il carteggio milanese dell'Archivio Datini di Prato, Firenze, Opuslibri, 1994.

Galoppini, Laura, Mercanti toscani e Bruges nel tardo Medioevo, Pisa, PLUS, 2009.

Goldthwaite, Richard A., The economy of Renaissance Florence, Baltimore, The Johns Hopkins - University Press, 2009.

Goldthwaite, Richard A.; Settesoldi, Enzo; Spallanzani, Marco, Due libri mastri degli Alberti. Una grande compagnia di Calimala 1348-1358, Firenze, Cassa di Risparmio di Firenze, 1995.

Mandich, Giulio, Per una ricostruzione delle operazioni mercantili e bancarie della compagnia Covoni, in Sapori, Armando (ed.), Libro giallo della compagnia dei Covoni, Milano, Cisalpino, 1970, pp. XCIX-CCXXIII.

Manni, Paola, Il libro del dare e dell'avere dei figli di Stefano Soderini (1306-1325), "Studi di Filologia Italiana" 37 (1978), pp. 67-155.

Manni, Paola, Frammenti d'un libro di conti in volgare pistoiese della prima metà del Dugento, "Studi Linguistici Italiani" 8 (1982), pp. 53-104.

Manni, Paola (ed.), Testi pistoiesi della fine del Dugento e dei primi del Trecento, Firenze, Accademia della Crusca, 1990.

Melis, Federigo, Storia della Ragioneria. Contributo alla conoscenza e interpretazione delle fonti più significative della storia economia, Bologna, Zuffi, 1950.

Melis, Federigo, Aspetti della vita economica medievale. Studi nell'Archivio Datini di Prato, vol. I, Siena, Monte dei Paschi, 1962.

Melis, Federigo, Documenti per la storia economica dei secoli XIII-XVI, Firenze, Olschki, 1972.

Melis, Federigo, Origini e sviluppi delle assicurazioni in Italia (secoli XIV-XVI), vol. I, Le fonti, Roma, Istituto Nazionale delle Assicurazioni,1975.

Melis, Federigo, Note di storia della banca pisana nel Trecento, in Melis, Federigo, La banca pisana e le origini della banca moderna (M. Spallanzani ed.), Firenze, Le Monnier, 1987, pp. 55-293.

Melis, Federigo, L'azienda nel Medioevo, (M. Spallanzani ed.), Firenze, le Monnier, 1991.

Melis, Federigo, Osservazioni preparatorie al bilancio nei conti della compagnia Farolfi, nel 1300, in Melis, Federigo, L'azienda nel Medioevo, (M. Spallanzani ed.), Firenze, Le Monnier, 1991, pp. 227-238.

ANUARIO de Estudios MEdievales, 42/2, julio-diciembre 2012, pp. 867-880

ISSN 0066-5061, doi:10.3989/aem.2012.42.2.11 
Molho, A.; Sznura, F. (eds.), Brighe, affanni, volgimenti di stato. Le ricordanze quattrocentesche di Luca di Matteo di messer Luca dei Firidolfi da Panzano, Firenze, Sismel - Edizioni del Galluzzo, 2010.

Mueller, Reinhold C., The Venetian money market: banks, panics, and the public debt, 1200-1500, Baltimore, The Johns Hopkins University Press, 1997.

Monaci, Ernesto, Crestomanzia italiana dei primi secoli, Città di Castello (Perugia), Lapi, 1912.

Nigro, Giampiero, Mercanti in Maiorca. Il carteggio datiniano dall'isola, 1387-1396, Firenze, Le Monnier, 2003.

Nigro, Giampiero (ed.), Francesco di Marco Datini. L'uomo il mercante, Firenze, Fondazione Istituto Internazionale di Storia Economica "F. Datini" - Firenze University Press, 2010.

Orlandi, Angela (ed.), Mercanzie e denaro. La corrispondenza datiniana tra Valencia e Maiorca, Valencia, Universitat de Valencia, 2008.

Paoli, Cesare, Documenti di Ser Ciappelletto, "Giornale Storico della Letteratura Italiana" 3/5 (1885), pp. 329-369.

Piccinni, Gabriella, Libri di contabilità privata e di memorie in Siena: considerazioni in merito all'esistenza, alla conservazione e alla scomparsa (XIII-XV secolo), "Bullettino Senese di Storia Patria" 115 (2008), pp. 164-198.

Piccinni, Gabriella, Sede pontificia contro Bonsignori di Siena. Inchiesta intorno a un fallimento bancario, in Rigon, A.; Veronese, F. (eds.), L'età dei processi. Inchieste e condanne tra politica e ideologia, Atti del convegno di studi (Ascoli 2007), Roma, Istituto Storico Italiano per il Medio Evo, 2009, pp. 213-246.

Piccinni, Gabriella, Il Banco dell'ospedale di Santa Maria della Scala e il mercato del denaro nella Siena del Trecento, Pisa, Pacini, 2012.

Santini, Piero, Frammenti di un Libro di banchieri fiorentini scritti in volgare nel 1211, "Giornale Storico della Letteratura Italiana" 5/10 (1887), pp. 161-196.

Sapori, Armando (ed.), I libri di commercio dei Peruzzi, Milano, Treves, 1934.

Sapori, Armando (ed.), I libri della ragione bancaria dei Gianfigliazzi, Milano, Garzanti, 1946.

Sapori, Armando, La compagnia dei Frescobaldi in Inghilterra, Firenze, Olschki, 1947.

Sapori, Armando (ed.), I libri degli Alberti del Giudice, Milano, Garzanti, 1952.

Sapori, Armando, Una compagnia di prestatori fiorentini ad Avignone nella prima metà del Trecento, in Sapori, Armando, Studi di storia economica, Firenze, Sansoni, 1955-1967, vol. III, pp. 101-119.

Sapori, Armando (ed.), Libro giallo della compagnia dei Covoni, Milano, Cisalpino, 1970.

Sapori, Armando, Premessa, in Libro giallo della compagnia dei Covoni, Milano, Cisalpino, 1970, pp. IX-XLIX.

Schiaffini, Alfredo (ed.), Testi fiorentini del Dugento e dei primi del Trecento, Firenze, Sansoni, 1926.

Serianni, Luca (ed.), Testi pratesi della fine del Dugento e dei primi del Trecento, Firenze, Accademia della Crusca, 1977.

Tognetti, Sergio, "Fra li compagni palesi et li ladri occulti". Banchieri senesi del Quattrocento, "Nuova Rivista Storica" 88 (2004), pp. 27-101.

Tognetti, Sergio, I mercanti-banchieri fiorentini e il ruolo di Venezia come piazza finanziaria europea nel tardo Medioevo. In margine al lavoro di R. C. Mueller, "Archivio Storico Italiano" 157 (1999), pp. 351-356.

Vitale, Marcella, Il quaderno di ricordi di messere Filippo Cavalcanti (1290-1324), "Studi di Filologia Italiana" 29 (1971), pp. 5-112.

Fecha de recepción del artículo: febrero 2011

Fecha de aceptación y versión final: octubre 2011

ANUARIO DE Estudios MEdievales, 42/2, julio-diciembre 2012, pp. 867-880

ISSN 0066-5061, doi:10.3989/aem.2012.42.2.11 